\title{
OBTENCIÓN DE ALCOHOL A PARTIR DE LA FERMENTACIÓN ANAEROBIA DEL MOSTO DE UVA
}

\section{OBTAINING ALCOHOL FROM THE ANAEROBIC FERMENTATION OF THE MUST OF GRAPE}

\author{
Alcívar-Bravo Andrés ${ }^{1 *}$; Barreiro-Cobeña Jandry²; Navia-Mendoza Jennifer; \\ Velásquez-Bazurto Selena ${ }^{4}$; Vinces-Muñoz Walker ${ }^{5}$
}

1,2,3,4,5Estudiantes de Ingeniería Química de la Facultad de Ciencias Matemáticas, Físicas y Químicas, Universidad Técnica de Manabí. Área de Tecnología, Programa de Ingeniería Química, Ingeniería de los Bioprocesos. Portoviejo, Ecuador.

*Correo: aalcivar3448@utm.edu.ec

\begin{abstract}
Resumen
Este trabajo tiene como objetivo principal obtener etanol mediante fermentación alcohólica aplicando procesos biotecnológicos; debido a su alto contenido en azucares se emplea el mosto de uva como sustrato y la levadura Saccharomyces cerevisiae como agente biológico para completar la fermentación, debido a que, este microorganismo ha sido probado en investigaciones anteriores mostrando una elevada tolerancia al etanol. Se tuvo en cuenta variables como el $\mathrm{pH}$, tiempo de fermentación, los grados Brix, formación de biomasa, entre otros. Los rendimientos de etanol y biomasa fueron de $0,483 \mathrm{gg}^{-1}$ y $0,069 \mathrm{gg}^{-1}$, respectivamente, además se establecieron las dimensiones del reactor en la escala industrial asumiendo una producción de $30 \mathrm{~L}$ de etanol.
\end{abstract}

Palabras clave: etanol, fermentación, mosto de uva.

\begin{abstract}
This work has as main objective to obtain ethanol by means of alcoholic fermentation applying biotechnological processes; due to its high sugar content, grape must is used as a substrate and Saccharomyces cerevisiae yeast as a biological agent to complete the fermentation, because this microorganism has been tested in previous investigations showing a high tolerance to ethanol. Variables such as $\mathrm{pH}$, fermentation time, Brix degrees, biomass formation, among others, were taken into account. The yields of ethanol and biomass were $0.483 \mathrm{gg}^{-1}$ and $0.069 \mathrm{gg}^{-1}$ respectively; the dimensions of the reactor on the industrial scale were established assuming a production of $30 \mathrm{~L}$ of ethanol.
\end{abstract}

Keywords: ethanol, fermentation, grape must.

Información del manuscrito:

Fecha de recepción: 06 de mayo de 2019

Fecha de aceptación: 24 de junio de 2019

Fecha de publicación: 10 de julio de 2019 


\section{Introducción}

Desde miles de años se han usado procesos de fermentación realizados por el hombre, con la finalidad de conservar alimentos 0 producir bebidas con sabor, aroma y textura específicas, entre esos productos se encuentra el yogurt, queso, cerveza, vino, entre otros. El vino es una bebida obtenida por fermentación alcohólica en condiciones enológicas de variedades de Vitis vinífera únicamente; el cultivo de estas vides cubre actualmente el $90 \%$ de las superficies de los viñedos en el mundo, mientras que el $10 \%$ restante son variedades no viníferas utilizadas generalmente como frutas de mesa (Miño, Herrera, \& Martos, 2007).

El etanol es un compuesto químico, conocido como alcohol etílico que se da en condiciones normales de presión y temperatura como un líquido incoloro, es una sustancia psicoactiva, volátil que está presente en diversas bebidas fermentadas, siendo el principal tipo de alcohol presente en las bebidas alcohólicas, como el vino (alrededor de un $13 \%$ ), la cerveza (5\%), los licores (hasta un $50 \%$ ) o los aguardientes (hasta un $70 \%$ ) (Arosemena et al., 2016).
En 2017, la producción mundial de etanol fue de 102 mil millones de litros, siendo Estados Unidos el mayor productor (58\%), seguido de Brasil (26\%) (Suárez et al., 2016).

Las levaduras son microorganismos eucariotas, unicelulares clasificados como miembros del reino Hongos. Estos microorganismos, y en particular, Saccharomyces cerevisiae, son de gran importancia económica, social y para la salud en la cultura humana. Se han utilizado para producir bebidas alcohólicas (como cerveza y vino) y fermentar la masa de pan durante milenios. Hoy en día, se han descrito otros usos para $S$. cerevisiae, incluida la producción de bioetanol como combustible, mediante procesos de fermentación alcohólica (Suárez et al., 2016).

Por lo anteriormente mencionado; en el desarrollo de este plan investigativo se establece como objetivo de investigación y experimentación, obtener etanol a partir de la fermentación alcohólica del mosto de uva, mediante el empleo de Saccharomyces cerevisiae como agente biológico y determinar la cantidad de producto que se puede obtener a escala de 
laboratorio, así como el diseño del reactor.

\section{Materiales y métodos}

\subsection{Preparación del sustrato}

Para la fermentación alcohólica del vino de uva, se empleó uva procedente del mercado municipal del cantón Tosagua, como sustrato, se procedió a realizar la selección de las frutas de acuerdo a sus características físicas, es decir, que no presentasen anomalías visibles 0 que el tiempo de maduración se haya extendido de lo debido; posteriormente se dispusieron a lavar, prensar manualmente y filtrar mediante una malla metálica para separar la piel y las semillas. Los grados Brix iniciales fueron 22.2.

\subsection{Preparación del inóculo}

Se empleó la levadura Saccharomyces cerevisiae como agente biológico para el proceso fermentativo del mosto de uva, para ello se utilizó $0,2340 \mathrm{~g}$ que fue activada con $0,5 \mathrm{~L}$ de agua destilada a temperatura de $37^{\circ} \mathrm{C}$ a $40^{\circ} \mathrm{C}$ durante $30 \mathrm{~min}$. Esta suspensión de levadura fue utilizada como inóculo para el proceso fermentativo.

\subsection{Diseño experimental}

Se inocularon recipientes que contenían $1 \mathrm{~L}$ de mosto con la cantidad correspondiente del inóculo de Saccharomyces cerevisiae. A cada mosto se le agregó $1,8 \mathrm{~g} \mathrm{hL}^{-1}$ de sulfato de amonio (coadyuvante de fermentación alcohólica). Los envases se mantuvieron obturados con válvula de agua para producir condiciones de anaerobiosis, además se incorporó otro orificio con una válvula que permitió la toma de muestra diaria de los grados Brix. Se iniciaron simultáneamente todas las fermentaciones en cámara isotérmica de $24^{\circ} \mathrm{C}$, y en condiciones escazas de luz. Cada una de las experiencias se realizó por triplicado. La fermentación se da por concluida cuando el grado Brix sea constante durante 48 a 72 h.

\subsection{Grados Brix}

Los grados Brix son una variable fundamental del proceso fermentativo, estos se midieron diariamente para conocer si el desdoblamiento de los azúcares en alcohol y gas carbónico se está produciendo, así como también, verificar si al final de la fermentación queda azúcar residual para 
fermentar y realizar los ajustes correspondientes.

Las mediciones se realizaron mediante el uso de un refractómetro (Hoyos, et al., 2010).

\subsection{Determinación de etanol}

El alcohol producto de la fermentación alcohólica del mosto se determinó mediante el empleo de un refractómetro, este resultado se expresa en porcentaje volumétrico.

\subsection{Determinación de biomasa}

Las determinaciones de biomasa se realizan con la técnica de peso seco, las células se separan del líquido bien por filtración, posteriormente se proceden a secar en una estufa a $120^{\circ} \mathrm{C}$ durante 30 minutos. La principal desventaja de esta técnica es que su determinación incluye no sólo microorganismos activos sino microorganismos muertos, material inerte, polímeros extracelulares y materia orgánica adsorbida (Arnaiz, 2015).

\subsection{Determinación de dióxido de carbono}

El dióxido de carbono resultado de la fermentación se lo determinó mediante titulación con $\mathrm{NaOH}$ al
0,09N, para ello se empleó fenolftaleína como indicador.

En la determinación del contenido de biomasa se incluyó también la levadura empleada para la fermentación.

\subsection{Diseño del biorreactor}

Un biorreactor es un recipiente 0 sistema que mantiene un ambiente biológicamente activo. En algunos casos, un biorreactor es un recipiente en el que se lleva a cabo un proceso químico que involucra organismos 0 sustancias bioquímicamente activas derivadas de dichos organismos; para el diseño del correspondiente biorreactor a escala industrial se llevó a cabo una serie de operaciones asumiendo un volumen de etanol a obtener para establecer así las dimensiones del reactor, el tipo de impelente, la potencia y principalmente, el volumen de mosto a emplear para la obtención del mismo.

\section{Resultados y discusión}

El mosto de uva tiene un ${ }^{\circ}$ Brix inicial de 16,8 , para aumentar su contenido de azúcar se añadieron $227 \mathrm{~g}$ de azúcar fermentable teniendo como resultado un Brix inicial de 22,2. 
Los valores de $\mathrm{pH}$ también fueron los indicados para iniciar las fermentaciones con uvas de variedades viníferas, el $\mathrm{pH}$ inicial del mosto fue 4,6, siendo el rango entre 3 y 5 el recomendado para variedades de Vitis vinífera (Rosier, 2008).

Tabla 1.

Datos de consumo de sustrato y valores de $\mathrm{pH}$

\begin{tabular}{|c|c|c|}
\hline Tiempo & ${ }^{\circ}$ Brix & pH \\
\hline 0 & 22.2 & 4.5 \\
\hline 1 & 20.0 & 4.3 \\
\hline 2 & 17.4 & 4.2 \\
\hline 3 & 11.67 & 4 \\
\hline 4 & 9 & 3.9 \\
\hline 5 & 6 & 3.7 \\
\hline 6 & 5.17 & 3.6 \\
\hline 7 & 5 & 3.5 \\
\hline
\end{tabular}

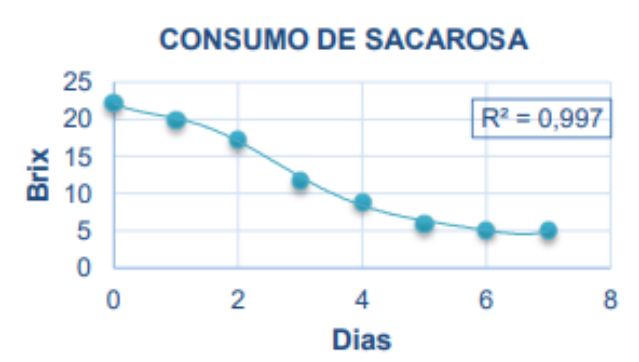

Figura 1. Cinética de consumo del sustrato

Los grados brix y el contenido de alcohol fueron determinados utilizando refractómetros, con lo cual se obtiene que los grados brix descendieron a $5^{\circ}$ brix, el contenido de alcohol fue de $12 \% \mathrm{v} / \mathrm{v}$. El $\mathrm{CO}_{2}$ producido durante los días de fermentación se determinó mediante titulación con $\mathrm{NaOH}$ al $0,09 \mathrm{~N}$ usando como indicador fenolftaleína, teniendo $0,244 \mathrm{~mol}$ de $\mathrm{CO}_{2}$ formado durante la reacción.

El rendimiento experimental del proceso en función de la producción de etanol respecto al consumo de sacarosa fue 0,483 ; mientras que el rendimiento teórico fue 0,5384 . La tabla 2 muestra el balance de materiales del proceso químico.

Tabla 2.

Balance de materiales

\begin{tabular}{|l|c|c|c|c|}
\hline Componente & Ingresa & Consume & Forma & Sale \\
\hline $\mathrm{C}_{12} \mathrm{H}_{22} \mathrm{O}_{11}$ & 234,26 & 79.49 & -- & 54.77 \\
\hline $\mathrm{NH}_{3}$ & 1.80 & 1.74 & -- & 0.06 \\
\hline $\mathrm{CH}_{1.8} \mathrm{O}_{0.5} \mathrm{~N}_{0.2}$ & -- & -- & 23.79 & 23.79 \\
\hline $\mathrm{CO}_{2}$ & -- & -- & 10.84 & 10.85 \\
\hline $\mathrm{H}_{2} \mathrm{O}$ & 765.74 & -- & 60.20 & 825.94 \\
\hline $\mathrm{C}_{2} \mathrm{H}_{6} \mathrm{O}$ & -- & -- & 88.35 & 88.35 \\
\hline
\end{tabular}

En la actualidad se han realizado estudios de cinética para la producción de etanol empleando la levadura S. Cerevisiae, donde se han registrado valores de $50,5 \mathrm{~g} / \mathrm{L}$ a las $24 \mathrm{~h}$, partiendo de $100 \mathrm{~g} / \mathrm{L}$ con un rendimiento del $97,2 \%$, en condiciones de fermentación, que han permitido conocer el tiempo en el que se consume el sustrato, así como la producción de etanol (Suárez, Garrido, \& Guevara, 2016).

A nivel estequiométrico, la secuencia de transformaciones para degradar la glucosa hasta dos moléculas de alcohol y dos moléculas de bióxido de carbono es un proceso muy complejo, pues al mismo tiempo la 
levadura utiliza la glucosa y nutrientes adicionales para reproducirse. Para evaluar esta transformación, se usa el rendimiento biomasa/producto y el rendimiento producto/ sustrato (Boudarel, 1984).

El rendimiento experimental obtenido al finalizar el proceso fermentativo fue de $0,483 \mathrm{gg}^{-1}$, este valor se encuentra bastante cercano al rendimiento teórico estequiométrico establecido y cuantificado por Gay Lussac, mismo que establece que para la transformación de glucosa en etanol se obtienen $0,511 \mathrm{~g}$ de etanol y 0,489 $\mathrm{g}$ de $\mathrm{CO}_{2}$ por $1 \mathrm{~g}$ de glucosa. En la realidad es difícil lograr este rendimiento, porque como se señaló anteriormente, la levadura utiliza la glucosa para la producción de otros metabolitos. El rendimiento experimental varía entre 90\% y 95\% del teórico, es decir, de 0,469 a 0,485 $\mathrm{g} / \mathrm{g}$. Los rendimientos en la industria varían entre 87 y $93 \%$ del rendimiento teórico (Vázquez \& Dacosta, 2007). En base a los resultados obtenidos se determinó que el flujo es de régimen turbulento y para lograr la homogenización de todo el caldo fermentativo es necesario emplear 88,35 $\mathrm{gr}$ de etanol; dicho proceso requiere de una velocidad de agitación de 1,67s ${ }^{1}$ y una potencia de $5100,52 \mathrm{~kW}$, es decir se trata de un proceso exotérmico, esto significa que la reacción está cediendo o liberando calor a los alrededores, por lo que aumenta su energía interna. Finalmente, es importante, controlar el tiempo de fermentación, puesto que, si el período es menor, la fermentación será incompleta y se desperdicia una considerable cantidad de alcohol, porque en el mosto queda todavía azúcar fermentable. Así mismo, el tiempo excesivo conduce a la pérdida de alcohol por formación de ácido acético y por evaporación.

\section{Conclusiones}

Se obtuvo un rendimiento de etanol a partir del mosto de uva de 0,49gg1 , obteniéndose un $90 \%$ de etanol según los resultados teóricos presentados por Gay Lusac, concluyendo así que el mosto de uva es un sustrato que presenta condiciones óptimas para llevar a cabo la formación de alcohol etílico a partir de un inóculo compuesto por Saccharomyces cerevisiae. 


\section{Agradecimientos}

A nuestra alma mater Universidad

Técnica de Manabí por permitirnos desenvolver ampliamente en el ámbito estudiantil. Especial agradecimiento al Ing. Ernesto Rosero PhD, catedrático de Ingeniería de los Bioprocesos, por contribuir con sus conocimientos de manera profesional en todos los aspectos relacionados con el presente artículo.

\section{Bibliografía}

Arnaiz, C. (2015). Determinación de la biomasa en procesos biológicos. Tecnología del agua, 45-52.

Arosemena, R., Castillo, A., Castillo, M., Castillo, V., Chen, R., Fuentes, A., \& Jaramillo, B. (2016). Obtención de etanol a base de la savia de la palma de corozo Attalea Butyracea. Universidad Tecnológica de Panamá, Ingeniería Industrial.

Boudarel, M. (1984). Contribution á l'étude de la Fermentation Alcoolique á partir de jus de Betteraves avec. Saccharomyces cerevisiae.
Thèse de Doctorat, Université de Dijon, Francia.

Hoyos, J. L., Urbano, F. E., Villada, H. S., Mosquera, S. A., \& Navia, D. P. (2010). Determinación de parámetros fermentativos para la formulación y obtención de alcohol de vino de naranja (Citrus sinensis). SCIELO.org, 8(1), 27-30.

Miño, J., Herrera, J., \& Martos, M. (2007). Micro vinificación tipo blush de uvas misioneras., VI Congreso Científico y Tecnológico de la FCEQyN. UNaM, Posadas Misiones Argentina, 367-370.

Rosier, J. P. (2008). Manual de elaboração de vinho para pequeñas cantinas., 2 ed. Florianópolis: EPAGRI. 72.

Suárez, C., Garrido, N., \& Guevara, C. (2016). Levadura Saccharomyces cerevisiae y la producción de alcohol. ICIDCA.

Vázquez, H., \& Dacosta, O. (2007). Fermentación alcohólica: Una opción para la producción de energía. Ingeniería. Investigación y Tecnología, VIII (4), 249- 259. 